\title{
Is switching to an oral antibiotic regimen safe after 2 weeks of intravenous treatment for primary bacterial vertebral osteomyelitis?
}

\author{
Baharak Babouee Flury ${ }^{1 *}$, Luigia Elzi ${ }^{1}$, Marko Kolbe², Reno Frei ${ }^{3}$, Maja Weisser ${ }^{1}$, Stefan Schären ${ }^{4}$, \\ Andreas F Widmer ${ }^{1}$ and Manuel Battegay ${ }^{1}$
}

\begin{abstract}
Background: Vertebral osteomyelitis (VO) may lead to disabling neurologic complications. Little evidence exists on optimal antibiotic management.

Methods: All patients with primary, non-implant VO, admitted from 2000-2010 were retrospectively analyzed. Patients with endocarditis, immunodeficiency, vertebral implants and surgical site infection following spine surgery were excluded. Persistence of clinical or laboratory signs of inflammation at 1 year were defined as treatment failure. Logistic regression was used to estimate the odds ratios (OR) of switch to an oral regimen after 2 weeks.

Results: Median antibiotic treatment was 8.1 weeks in 61 identified patients. Switch to oral antibiotics was performed in $72 \%$ of patients after a median intravenous therapy of 2.7 weeks. Switch to oral therapy was already performed after two weeks in 34\% of the patients. A lower CRP at 2 weeks was the only independent predictor for switch to oral therapy (OR 0.7, 95\% confidence interval 0.5-0.9, $p=0.041$, per $10 \mathrm{mg} / \mathrm{l}$ increase). Staphylococcus aureus was the most frequently isolated microorganism (21\%). Indications for surgery, other than biopsy, included debridement with drainage of epidural or paravertebral abscess (26 patients; $42 \%)$, and CT - guided drainage (3 patients).

During the follow-up, no recurrences were observed but 2 patients died of other reasons than $\mathrm{VO}$, i.e. the 1 year intention to treat success rate was $97 \%$.

Conclusions: Cure rates for non-implant VO were very high with partly short intravenous and overall antibiotic therapy. Switching to an oral antibiotic regimen after two weeks intravenous treatment may be safe, provided that CRP has decreased and epidural or paravertebral abscesses of significant size have been drained.
\end{abstract}

Keywords: Vertebral osteomyelitis, Switch to oral therapy, Antibiotic management

\section{Background}

The incidence of vertebral osteomyelitis (VO) is increasing, primarily affecting elderly patients and those with comorbidities [1,2]. Complications of VO, i.e. epidural, paravertebral or psoas abscesses, which result from direct seeding of the microorganism in different compartments [1], may lead to longer hospital stay and higher mortality. To date, there are no consistent data from randomized controlled trials to guide the optimal duration and appropriate route of antibiotic therapy. Recommendations

\footnotetext{
* Correspondence: baharak.babouee@phe.gov.uk

'Division of Infectious Diseases and Hospital Epidemiology, University Hospital, Petersgraben 4, Basel 4031, Switzerland

Full list of author information is available at the end of the article
}

for the length of antibiotic therapy vary considerably [3-5], with suggestion of parenteral treatment between 3 and 8 weeks [6-9]. The mean treatment duration in a French multicentre observational prospective study, involving 110 patients, was 14.7 weeks, with minimum length ranging from 6 to 12 weeks according to the treating centre [6]. Other observational studies show distinctive differences in recurrence rates for treatment duration of less than 4 weeks (>14\%), 6 weeks (10\%), 8 weeks (>15\%) compared to longer than 12 weeks (3.9\%) [10].

We retrospectively investigated patients with primary VO in a tertiary care hospital during a 10-year period with the aim to assess predictors of switching from intravenous

\section{Ciomed Central}

(c) 2014 Babouee Flury et al.; licensee BioMed Central Ltd. This is an Open Access article distributed under the terms of the Creative Commons Attribution License (http://creativecommons.org/licenses/by/2.0), which permits unrestricted use, distribution, and reproduction in any medium, provided the original work is properly credited. 
to an oral antibiotic regimen and to investigate the clinical outcome dependent on different treatment durations.

\section{Methods}

\section{Study population and design}

This study is a retrospective analysis of all patients diagnosed with VO, seen by an Infectious Disease specialist, at the University Hospital of Basel, Switzerland, between 2000 and 2010. The University Hospital of Basel is a tertiary 800-bed institution serving the northwestern part of Switzerland with a population of approximately half a million people.

Patients were selected from the Infectious Diseases patient database. Supplementary data collected by chart review included demographic characteristics, date of hospitalisation, co-morbidities (diabetes mellitus, cardiovascular disease, immunosuppression and renal insufficiency). Blood tests included creatinine, white blood cell count and C-reactive protein (CRP). Further data comprised the clinical presentation of $\mathrm{VO}$ such as back pain, fever, neurologic deficits at presentation (i.e. limb weakness, dysesthesia or sensory loss, retention of urine and radiculopathy), the presence of endocarditis, radiological images, microbiological tests (blood cultures, bone biopsies), antibiotic treatment regimen including route of administration (oral or intravenous) and duration and surgical procedures.

Diagnosis of VO was made if two of the following criteria were present: (1) clinical presentation compatible with VO [1] such as fever, back pain or neurological deficits, (2) compatible radiological images [11,12] and (3) identification [13] of pathogen by blood culture, needle biopsy or surgical biopsy. We excluded patients with endocarditis, as the recommended intravenous treatment is at least 4 weeks, surgical site infection following spine surgery because a 2-week intravenous antibiotic course after extensive debridement is generally considered to be adequate before switching to an oral regimen $[14,15]$ and spinal implants or tuberculous VO because of the longer treatment duration $[16,17]$.

\section{Outcome measures}

The physicians, caring for patients after discharge from hospital, were requested to provide follow-up data on the clinical course, laboratory parameters and treatment at 4 and 6 weeks and 1 year after diagnosis of VO.

Recovery was defined as disappearance of all clinical signs and symptoms of VO with no residual disability [18].

Treatment failure was defined as the persistence of clinical signs and symptoms of VO, i.e. fever, residual pain or neurological symptoms, or laboratory signs of inflammation with no other explanation, VO-related re-hospitalization, relapse or VO-related death. The outcome was evaluated after completing the prescribed antibiotic therapy and at 1 year by in-hospital data and a questionnaire sent to the treating physicians.

\section{Statistical analysis}

Basic demographic characteristics, co-morbidities, laboratory and microbiological parameters, surgical and antibiotic management were compared using the ${ }_{X}^{2}$ test or Fisher's exact test for categorical variables and the Mann-Whitney test for continuous variables. Logistic regression was used to estimate the odds ratios (OR) of switch to an oral regimen after 2 weeks.

All analyses were performed using STATA $^{\mathrm{m}}$ software version 11 for Windows (StataCorp, College Station, TX, USA). This study was approved by the local Ethics Committee (Ethische Komission beider Basel).

\section{Results}

\section{Study population}

Among 129 patients with VO, 68 patients were excluded because of spinal implants (25), endocarditis (19), surgical site infections (10) and missing follow-up data (14). The final analysis was performed on 61 patients. The median age was 65 years [interquartile range (IQR) 51-76] and most patients were male (61\%). Baseline characteristics are shown in Table 1.

\section{Clinical presentation and diagnosis of Vo}

Twenty-seven patients had no comorbidities, all other patients were reported to have more than 1 underlying medical disease, arterial hypertension being the most frequent (39\%) followed by coronary heart disease (25\%), diabetes mellitus (11\%) and renal impairment, i.e. creatinine clearance $<80 \mathrm{ml} / \mathrm{min}(8 \%)$. Back pain was by far the most common presenting symptom (93\%), followed by fever $(28 \%)$. Neurologic deficits at presentation were reported in $16 \%$ and abscess formation (i.e. epidural or paravertebral) was found in $51 \%$ of patients.

Staphylococcus aureus and coagulase-negative staphylococci were the most frequently isolated microorganisms, i.e. $21 \%$ and $17 \%$ respectively, followed by gram-negative bacteria (28\%), streptococci (20\%) and Propionibacterium acnes (5\%). In 8 patients no causing microorganism could be identified.

Diagnostic tools for bacteriologic diagnosis included blood cultures, computerized tomography (CT) - guided needle biopsy and open biopsy. In 49 patients blood cultures were performed. Of these, 25 (51\%) were positive with microorganisms regarded as causative pathogens for the VO. Biopsies (CT-guided and open) were performed in 50 patients (82\%) yielding a causative bacterium in $92 \%$.

Radiological techniques for the diagnosis of $\mathrm{VO}$ included computerized tomography (CT) with contrast 
Table 1 Baseline characteristics of the study population ( $\mathrm{n}=61)$

\begin{tabular}{|c|c|c|c|}
\hline \multirow{2}{*}{\multicolumn{2}{|c|}{ Characteristic }} & \multicolumn{2}{|c|}{ All patients } \\
\hline & & $\mathrm{n}^{*}$ & $\%^{*}$ \\
\hline \multicolumn{2}{|l|}{ Males } & 37 & 61 \\
\hline \multicolumn{2}{|c|}{ Median age, IQR } & 65 & $51-76$ \\
\hline \multicolumn{2}{|c|}{ Median BMI, IQR } & 25 & $21-28$ \\
\hline \multicolumn{2}{|c|}{ Arterial hypertension } & 24 & 39 \\
\hline \multicolumn{2}{|c|}{ Diabetes mellitus } & 7 & 11 \\
\hline \multicolumn{2}{|c|}{ Coronary heart disease } & 15 & 25 \\
\hline \multicolumn{2}{|c|}{ Renal impairment (clearance $<80 \mathrm{ml} / \mathrm{min}$.) } & 5 & 8 \\
\hline \multicolumn{2}{|l|}{ Fever $\left(>38^{\circ} \mathrm{C}\right)$} & 17 & 28 \\
\hline \multicolumn{2}{|l|}{ Back pain } & 57 & 93 \\
\hline \multicolumn{2}{|c|}{ Neurologic al deficits } & 10 & 16 \\
\hline \multicolumn{2}{|l|}{ Epidural abscess } & 31 & 51 \\
\hline \multicolumn{2}{|c|}{ Median leucocytes count, IQR $\left(10^{9} / \mathrm{I}\right)$} & 10.1 & $8.0-12.5$ \\
\hline \multicolumn{2}{|c|}{ Median C-reactive protein (mg/l) } & 100 & $62-186$ \\
\hline \multirow[t]{8}{*}{ Microorganism } & Staphylococcus aureus & 11 & 21 \\
\hline & Coagulase negative staphylococci & 9 & 17 \\
\hline & Streptococci spp. & 12 & 20 \\
\hline & Escherichia coli & 6 & 11 \\
\hline & Klebsiella spp. & 2 & 4 \\
\hline & Pseudomonas aeruginosa & 7 & 13 \\
\hline & Propionibacterium acnes & 3 & 6 \\
\hline & Other or unknown & 11 & 21 \\
\hline
\end{tabular}

*unless otherwise stated.

medium in 10 patients (16\%) and/or magnetic resonance tomography (MRI) in 58 patients (95\%).

\section{Management of vertebral osteomyelitis}

All patients received empirical antibiotic treatment for $\mathrm{VO}$ according to internal guidelines, after diagnostic procedures have been performed being amoxicillinclavulanate and ceftriaxone the most frequently used (Table 2). The empiric antibiotic therapy was retrospectively considered to be adequate in 47 patients $(83 \%)$ according to definite microbiological results. The median duration of antibiotic treatment was 57 days (8.1 weeks; IQR 44-83 days). Switch to an oral antibiotic regimen was performed in $72 \%$ of patients after a median intravenous therapy of 19 days (2.7 weeks; IQR 14-27 days). In 21 patients $(34 \%)$ the switch to oral therapy was performed after 2 weeks of intravenous treatment. The most frequently used oral antibiotic treatment was ciprofloxacin or another quinolone alone (35\%) and a combination of ciprofloxacin and rifampin (26\%).

Indications for surgery, other than biopsy, included debridement with drainage of the abscess in 26 of 31 patients with epidural or paravertebral abscess, whereas
Table 2 Antibiotic treatment and surgical management of 61 patients with primary spondylodiscitis

\begin{tabular}{|c|c|c|c|}
\hline \multirow[t]{2}{*}{ Characteristic } & & \multicolumn{2}{|c|}{ All patients } \\
\hline & & $\mathrm{n}^{*}$ & $\% *$ \\
\hline \multirow[t]{2}{*}{ Procedure } & Surgery & 26 & 43 \\
\hline & CT-guided drainage & 3 & 5 \\
\hline \multicolumn{2}{|c|}{ Adequate empirical antibiotic therapy } & 47 & 83 \\
\hline \multirow[t]{7}{*}{ Empirical therapy } & Amoxicillin-clavulanate & 29 & 48 \\
\hline & Flucloxacillin & 5 & 8 \\
\hline & Ceftriaxon & 9 & 15 \\
\hline & Piperacillin-tazobactam & 6 & 10 \\
\hline & Other beta-lactame antibiotics & 5 & 8 \\
\hline & Ciprofloxacin & 5 & 8 \\
\hline & Other & 2 & 3 \\
\hline \multicolumn{2}{|c|}{ Switch to oral antibiotic treatment } & 43 & 72 \\
\hline \multirow[t]{5}{*}{ Oral regimen } & Ciprofloxacin or other chinolone alone & 15 & 35 \\
\hline & Ciprofloxacin and clindamycin & 5 & 12 \\
\hline & Ciprofloxacin and rifampicin & 11 & 26 \\
\hline & Clindamycin alone & 3 & 7 \\
\hline & Other & 9 & 21 \\
\hline \multicolumn{2}{|c|}{ Antibiotic regimen containing rifampicin } & 15 & 25 \\
\hline \multicolumn{2}{|c|}{ Median duration of total antibiotic therapy, IQR (days) } & 57 & $44-83$ \\
\hline
\end{tabular}

*unless otherwise stated.

CT - guided drainage of the abscess was performed in 3 patients. In 2 patients no drainage or surgery was performed due to the very small size of epidural abscess.

\section{Predictors of switch to an oral antibiotic regimen after 2 weeks}

A univariate and multivariate analysis was performed. After adjustment for age, gender, comorbidity, fever and neurological deficits at diagnosis, microorganisms, abscesses (i.e. epidural or paravertebral), surgery and laboratory parameters, correct empirical antibiotic therapy, as well as antibiotic regimen containing rifampicin, lower CRP at 2 weeks compared to baseline CRP was the only independent predictor of switching to an oral antibiotic regimen after 2 weeks (OR 0.7, 95\% CI 0.5-0.9, p = 0.041, per $10 \mathrm{mg} / \mathrm{l}$ increase).

\section{Outcome}

A complete follow-up was available for 61 patients. During the follow-up, 2 patients died of metastasizing colorectal and bronchial carcinoma, 47 and 51 days after diagnosis of $\mathrm{VO}$, i.e. the 1-year success rate in an intention to treat analysis was $97 \%$ and there were no reported re-hospitalisations and/or treatment failures because of $\mathrm{VO}$. 


\section{Discussion}

Our study, involving 61 immunocompentent patients with primary vertebral osteomyelitis, illustrates that switching to an oral antibiotic regimen after 2 weeks of intravenous therapy may be safe, provided that symptoms have improved, epidural or paravertebral abscess has been drained and C-reactive protein levels have decreased. Importantly, our results do not extend to patients with endocarditis, surgical site infection and vertebral implants.

The duration of intravenous therapy has not been established so far. Several studies described successful switch to oral antibiotics after 10 days, using oral agents with a high bio-availability and tissue penetration, i.e. fluorquinolones, rifampin, fusidic acid and clindamycin [19], after endocarditis had been excluded $[2,20]$. In the study of Beronius et al. [20], however, the median duration of oral antibiotic therapy after a short parenteral therapy was 179 days (range 46-640 days), which is much longer than the therapy duration in our patient collective. This might be due to the fact that $27 \%$ of the patients in the above mentioned study had tuberculous VO.

Thirty-five percent of our patients were treated with fluorquinolones alone and $26 \%$ with a combination of a fluorquinolone and rifampin, all with good outcome, i.e. cure. Fluorquinolones are bactericidal drugs and thereby allow an early switch to the oral route. In a randomized clinical study, the combination of an oral fluorquinolone and rifampin in case of staphylococcal bone or joint infections, resulted in cure rates that were similar to those with the standard intravenous therapy [21].

In our study, a lower CRP at 2 weeks was the only independent predictor of switching to an oral antibiotic regimen. Serum CRP level is closely related with the clinical response to therapy and is therefore the preferred marker of the course of infection [22]. Criteria for discontinuation of antimicrobial therapy include resolution of clinical symptoms as well as normalization of CRP $[6,18]$. It has been proposed that a weekly decrease in CRP by $50 \%$ represents treatment response [23]. Lack of improvement in symptoms such as continued fever and no reduction in pain or a persistently elevated CRP above $30 \mathrm{mg} / \mathrm{l}$ are predictors of treatment failure [22,24]. In our study, the early switch to oral antibiotic therapy, i.e. after two weeks, if above mentioned criteria are met, does not seem to be associated with adverse outcome.

In the present study $51 \%$ of the patients were found to have epidural and/or paravertebral abscess formation. This high number can be explained by the fact that MRI and/or CT scans were systematically performed in all patients to establish the diagnosis of VO. All, but two of the patients (93\%) with abscesses required surgery. This rate is much higher than reported in other studies $[3,25]$. All patients with abscess formation were treated according to our internal guidelines which are in line with published procedures [1]. This implies surgical drainage and/or debridement and is likely to have contributed to the high number of surgical intervention in case of abscess formation.

Open surgical decompression combined with intravenous antibiotic treatment has long been considered the cornerstone of management for spinal epidural abscess [26]. Following this management may be associated with a shorter duration of intravenous therapy in our study.

The aetiological microorganism could be isolated with blood cultures in 51\% and with CT-guided or open biopsy in $75 \%$ of patients. Staphylococcus aureus and gram negative microorganisms were the most common causative microorganisms in our patient group. This is in line with previous studies where Staphylococcus aureus was the most common isolated organism followed by gramnegative bacilli, E.coli being the predominant agent $[1,13,27]$. Despite exclusion of endocarditis and spinal implants, as well as surgical site infections, $17 \%$ of the microorganisms detected were coagulase - negative staphylococci (CoNS) proven by bone biopsies. In these patients, biopsies were taken before empiric antibiotic therapy was initiated and CoNS-targeted antibiotic therapy led to resolution of symptoms. CoNS are common pathogens in cases of sternal osteomyelitis following median sternotomy [28] and are associated with intra-cardiac device related bacteraemia $[29,30]$. In contrast, they rarely cause osteomyelitis in the absence of bone devices or in patients without profound immunosuppression [31]. Our findings however indicate that CoNS might be more often found as the causative organisms in $\mathrm{VO}$ even without foreign bodies or endocarditis. We speculate that sclerosis of the bone in ageing patients may predispose to infections with CoNS.

In our study we had excellent outcome results. This might be due to the reason, that patients with endocarditis, immunodeficiency, vertebral implants and surgical site infection following spine surgery had been excluded. Latter mentioned patients tend to have more complications. E.g. recurrent bacteraemia in case of endocarditis was independently associated with relapse [18]. Another reason for the excellent outcome might be the high rate of drainage in case of abscesses. In the above mentioned study of Mc Henry et al. [18] surgical treatment resulted in recovery or improvement in $79 \%$ of patients.

A third reason for the excellent outcome is likely the fact that in every case an infectious disease specialist was involved and antibiotic therapy had continuously been adapted. In this respect the very low prevalence of MRSA may have helped the excellent outcome.

\section{Limitations and strengths}

Our results may have a limited generalizability as the study was performed in a single centre. However, the 
study was conducted over an extended period of time with consistent results. Limited generalizability is also given due to the local epidemiology with a very low prevalence of methicillin-resistant $S$. aureus (MRSA) of 5-7\% (data not shown). Due to frequently co-occuring resistance to rifampin in MRSA, one of the most important oral treatment options is futile in many instances. In our institution, we were able to switch to oral antibiotics in a large part of patients. A second limitation of the study is its retrospective study design; we cannot exclude a selection bias of patients who received a shorter intravenous antibiotic treatment. Selection bias may have occurred because physicians tend to switch earlier to an oral regimen if the patient has improved, so that switched patients may be those with better prognosis and/or more limited disease. On the other hand physicians may have had prolonged the intravenous therapy in patients which were not responding optimally to the therapy. Finally, the patient number did not allow for strong multivariate analyses, e.g. we could not define the level of C-reactive protein, above which intravenous therapy should be prolonged.

Our study has also strengths: First, the study covers a long time period with consistent results. Noteworthy, to the best of our knowledge, this is the first retrospective study of a homogenous patient collective (i.e. with primary vertebral osteomyelitis) after exclusion of patients with endocarditis, immunodeficiency, spinal implants and surgical site infection following spine surgery. Furthermore, the patient population was worked up in a meticulous way demonstrated by high rates of microbiological diagnoses and high rates of diagnosed abscesses.

\section{Conclusion}

Our results suggest that switching to an oral antibiotic regimen after two weeks of intravenous therapy is safe in immunocompetent patients for primary non-implant vertebral osteomyelitis if epidural or paravertebral abscesses have been drained and if an oral antibiotic therapy with documented susceptibility, high bio-availability and bactericidal activity is available. Our results should be confirmed by a prospective randomized controlled trial.

\section{Competing interests}

The authors declare that they have no competing interests.

\section{Authors' contribution}

BBF has made substantial contributions to conception of the study and interpretation of data and drafted the manuscript. LE has designed the study, performed statistical analysis, interpreted the data and helped to draft the manuscript. MK has collected the data and contributed in the conception of the study. RF has carried out the microbiological exams and contributed in data collection. MW has made substantial contribution to acquisition of data and has revised the manuscript critically. SS has performed the surgical procedures and revised the manuscript. AW has made substantial contribution in data interpretation. MB participated in the design and coordination of the study helped to draft the manuscript. All authors read and approved the final manuscript.

\section{Acknowledgements}

This study was presented in part at the 22nd European Congress of Clinical Microbiology and Infectious Diseases (ECCMID) in London, Great Britain, 2012 (Poster 2219).

\section{Funding}

This study has been supported by the Stiftung Forschung Infektionskrankheiten, Basel (B.BF).

\section{Author details}

'Division of Infectious Diseases and Hospital Epidemiology, University Hospital, Petersgraben 4, Basel 4031, Switzerland. ${ }^{2}$ Medical Faculty, University of Basel, Basel, Switzerland. ${ }^{3}$ Division of Clinical Microbiology, University Hospital Basel, Basel, Switzerland. ${ }^{4}$ Department of Orthopedic Surgery, University Hospital of University Basel, Basel, Switzerland.

Received: 22 August 2013 Accepted: 17 April 2014

Published: 27 April 2014

\section{References}

1. Zimmerli W: Clinical practice. Vertebral osteomyelitis. N Engl J Med 2010, 362(11):1022-1029.

2. Gouliouris T, Aliyu SH, Brown NM: Spondylodiscitis: update on diagnosis and management. J Antimicrob Chemother 2010, 65 Suppl 3:iii11-24.

3. Roblot F, Besnier JM, Juhel L, Vidal C, Ragot S, Bastides F, Le Moal G, Godet C, Mulleman D, Azais I, Becq-Giraudon B, Choutet P: Optimal duration of antibiotic therapy in vertebral osteomyelitis. Semin Arthritis Rheum 2007, 36(5):269-277.

4. Livorsi DJ, Daver NG, Atmar RL, Shelburne SA, White AC Jr, Musher DM: Outcomes of treatment for hematogenous Staphylococcus aureus vertebral osteomyelitis in the MRSA ERA. J Infect 2008, 57(2):128-131.

5. Musher DM, Thorsteinsson SB, Minuth JN, Luchi RJ: Vertebral osteomyelitis. Still a diagnostic pitfall. Arch Intern Med 1976, 136(1):105-110.

6. Legrand E, Flipo RM, Guggenbuhl P, Masson C, Maillefert JF, Soubrier M, Noel E, Saraux A, Di Fazano CS, Sibilia J, Goupille P, Chevalie X, Cantagrel A, Conrozier T, Ravaud P, Lioté F: Management of nontuberculous infectious discitis. treatments used in 110 patients admitted to 12 teaching hospitals in France. Joint Bone Spine 2001, 68(6):504-509.

7. Hadjipavlou AG, Mader JT, Necessary JT, Muffoletto AJ: Hematogenous pyogenic spinal infections and their surgical management. Spine 2000, 25(13):1668-1679.

8. Osenbach RK, Hitchon PW, Menezes AH: Diagnosis and management of pyogenic vertebral osteomyelitis in adults. Surg Neurol 1990, 33(4):266-275

9. Liebergall M, Chaimsky G, Lowe J, Robin GC, Floman Y: Pyogenic vertebral osteomyelitis with paralysis. Prognosis and treatment. Clin Orthop Relat Res 1991, 269:142-150

10. Grados F, Lescure FX, Senneville E, Flipo RM, Schmit JL, Fardellone P: Suggestions for managing pyogenic (non-tuberculous) discitis in adults. Joint Bone Spine 2007, 74(2):133-139.

11. Teman AJ: Spinal epidural abscess. Early detection with gadolinium magnetic resonance imaging. Arch Neurol 1992, 49(7):743-746.

12. Palestro CJ, Love C, Miller T: Infection and musculoskeletal conditions: imaging of musculoskeletal infections. Best Pract Res Clin Rheumatol 2006, 20(6):1197-1218.

13. Mylona E, Samarkos M, Kakalou E, Fanourgiakis P, Skoutelis A: Pyogenic vertebral osteomyelitis: a systematic review of clinical characteristics. Semin Arthritis Rheum 2009, 39(1):10-17.

14. Sendi P, Zimmerli W: Antimicrobial treatment concepts for orthopaedic device-related infection. Clin Microbiol Infect 2012, 18(12):1176-1184.

15. Zimmerli W, Trampuz A, Ochsner PE: Prosthetic-joint infections. N Engl J Med 2004, 351(16):1645-1654.

16. Bass JB Jr, Farer LS, Hopewell PC, O'Brien R, Jacobs RF, Ruben F, Snider DE $\mathrm{Jr}$, Thornton G: Treatment of tuberculosis and tuberculosis infection in adults and children. American thoracic society and the centers for disease control and prevention. Am J Respir Crit Care Med 1994, 149(5):1359-1374.

17. Joint Tuberculosis Committee of the British Thoracic Society: Chemotherapy and management of tuberculosis in the United Kingdom: recommendations, 1998. Thorax 1998, 53(7):536-548. 
18. McHenry MC, Easley KA, Locker GA: Vertebral osteomyelitis: long-term outcome for 253 patients from 7 Cleveland-area hospitals. Clin Infect Dis 2002, 34(10):1342-1350.

19. Zeller V, Desplaces N: Antibiotherapy of bone and joint infections (Antibiothérapie des infections ostéoarticulaires à pyogènes chez l'adulte: principe et modalités). Rev Rhum 2006, 73:183-190.

20. Beronius M, Bergman B, Andersson R: Vertebral osteomyelitis in Goteborg, Sweden: a retrospective study of patients during 1990-95. Scand J Infect Dis 2001, 33(7):527-532.

21. Schrenzel J, Harbarth S, Schockmel G, Genne D, Bregenzer T, Flueckiger U, Petignat C, Jacobs F, Francioli P, Zimmerli W, Lew DP: A randomized clinical trial to compare fleroxacin-rifampicin with flucloxacillin or vancomycin for the treatment of staphylococcal infection. Clin Infect Dis 2004, 39(9):1285-1292.

22. Khan MH, Smith PN, Rao N, Donaldson WF: Serum C-reactive protein levels correlate with clinical response in patients treated with antibiotics for wound infections after spinal surgery. Spine J 2006, 6(3):311-315.

23. Legrand E, Massin P, Levasseur R, Hoppé E, Chappard D, Audran M: Stratégie diagnostique et principes thérapeutiques au cours des spondylodiscites infectieuses bactériennes. Rev Rhum 2006, 73:373-379.

24. Kowalski TJ, Berbari EF, Huddleston PM, Steckelberg JM, Osmon DR: Do follow-up imaging examinations provide useful prognostic information in patients with spine infection? Clin Infect Dis 2006, 43(2):172-179.

25. Rigamonti D, Liem L, Sampath P, Knoller N, Namaguchi Y, Schreibman DL, Sloan MA, Wolf A, Zeidman S: Spinal epidural abscess: contemporary trends in etiology, evaluation, and management. Surg Neurol 1999, 52(2):189-196. discussion 197 .

26. Siddiq F, Chowfin A, Tight R, Sahmoun AE, Smego RA Jr: Medical vs surgical management of spinal epidural abscess. Arch Intern Med 2004, 164(22):2409-2412

27. Cottle L, Riordan T: Infectious spondylodiscitis. J Infect 2008, 56(6):401-412.

28. Rupp ME, Archer GL: Coagulase-negative staphylococci: pathogens associated with medical progress. Clin Infect Dis 1994, 19(2):231-243. quiz 244-235.

29. Bucher E, Trampuz A, Donati L, Zimmerli W: Spondylodiscitis associated with bacteraemia due to coagulase-negative staphylococci. Eur J Clin Microbiol Infect Dis 2000, 19(2):118-120.

30. Le Moal G, Roblot F, Paccalin M, Sosner P, Burucoa C, Roblot P, Becq-Giraudon $B$ : Clinical and laboratory characteristics of infective endocarditis when associated with spondylodiscitis. Eur J Clin Microbiol Infect Dis 2002, 21(9):671-675.

31. Sapico FL: Microbiology and antimicrobial therapy of spinal infections Orthop Clin North Am 1996, 27(1):9-13.

doi:10.1186/1471-2334-14-226

Cite this article as: Babouee Flury et al:: Is switching to an oral antibiotic regimen safe after 2 weeks of intravenous treatment for primary bacterial vertebral osteomyelitis? BMC Infectious Diseases 2014 14.226.

\section{Submit your next manuscript to BioMed Central and take full advantage of:}

- Convenient online submission

- Thorough peer review

- No space constraints or color figure charges

- Immediate publication on acceptance

- Inclusion in PubMed, CAS, Scopus and Google Scholar

- Research which is freely available for redistribution 